\title{
ANÁLISE DO MEDO DE CAIR EM IDOSAS PRATICANTES DE EXERCíCIOS FÍSICOS E SEDENTÁRIAS
}

José Henrique Piedade Cardoso, Luana Martins de Paula, Silas de Oliveira Damasceno, Henrique Martins Ungri, Ronaldo Valdir Briani, Ana Caroline Rippi Moreno, Laís Manata Vanzela ${ }^{1}$, Franciele Marques Vanderlei, Giovana Gomes dos Santos, Bianca Yumie Eto, Claudia Regina Sgobbi Faria

Universidade Estadual Paulista "Júlio Mesquita Filho" - UNESP, Departamento de Fisioterapia, Presidente Prudente, SP. E-mail: zepiedade@hotmail.com

\section{RESUMO}

A incidência de quedas é elevada em idosos e o medo de cair está entre as decorrências mais universais de quedas nesta população. Nesse sentido, o estudo tem por objetivo analisar o equilíbrio e medo de quedas em idosas praticantes de atividade física e em sedentárias. Para isso, foram avaliadas 30 idosas do sexo feminino divididas em dois grupos: exercício terapêutico e sedentarismo. Ambos avaliados utilizando a Montreal Cognitive Assessment (MoCA), Falls Efficacy Scale (FES-I-Brasil) e Escala de equilíbrio de Tinetti. Os resultados demonstram diferença significativa na análise do medo de cair $(20,09 \pm 3,7$ vs $37,72 \pm 8,7 ; p=0,0003)$, equilíbrio e marcha $(27,77 \pm 0,5$ vs $18,40 \pm 3,9 ; p<0,0001)$ favor das idosas ativas. Já para as variáveis idade $(68,04 \pm 5,3$ vs $72,54 \pm 8,4 ; p=0,04)$ e cognição $(28,31 \pm 1,5$ vs $24,45 \pm 1,8 ; p=0,44)$, diferenças significativas não foram observadas. Desta forma conclui-se que idosas fisicamente ativas apresentam menor preocupação com a possiblidade de cair e melhores níveis de equilíbrio.

Palavras-chave: Idosas, quedas, medo de cair, funcionalidade.

\section{ANALYSIS OF THE FEAR OF FALL IN ELDERLY WOMEN PRACTICING PHYSICAL EXERCISES AND SEDENTARY}

\begin{abstract}
The incidence of falls is high in elderly and the fear of falls is between the universal occurences of falls in this population. In this sense, the study has by objective evaluate the balance and the fear of falls in elderly woman participants of physical activity and sedentaries. Were evaluated 30 ederly of feminine sex divided into two groups: therapeutic exercise and sedentarism. All groups were evaluated using the Montreal Cognitive Assessment (MoCA), Falls Efficacy Scale (FES-I-Brasil) and Tinetti Balance Scale. The results showed significative difference on fear of falls $(20.09 \pm 3.7$ vs $37.72 \pm 8.7 ; p=0.000)$, balance and march $(27.77 \pm 0.5$ vs $18.40 \pm 3.9 ; p<0.000)$ in favor to active elderly woman. For the variables age $(68.04 \pm 5.3$ vs $72.54 \pm 8.4 ; p=0.04)$ and cognition $(28.31 \pm 1.5$ vs $24.45 \pm 1.8 ; p=0.44)$, significative diferences were not observed. At this form, we conclude that physically active elderly woman presented less preocupation with the possibility of falls and better balance levels.
\end{abstract}

Keywords: Elderly, falls, fear of falling, functionality. 


\section{INTRODUÇÃO}

O Brasil, assim como em diversos países do mundo, está vivenciando uma transição demográfica marcada pelo envelhecimento populacional, e consequente crescimento da população idosa ${ }^{1}$. O envelhecimento é um processo dinâmico, fisiológico e progressivo no qual ocorrem diversas alterações psicológicas, sociais e biológicas ${ }^{2}$.

Dentre as alterações biológicas, destaca-se a redução das capacidades físicas como flexibilidade, força dos membros superiores e inferiores, agilidade, capacidade cardiorrespiratória e equilíbrio corporal, que influenciam no controle postural e está diretamente ligada à ocorrência de quedas ${ }^{2,3}$

As alterações sensoriais e neuromusculares também influenciam diretamente a estabilidade de indivíduos da terceira idade ${ }^{4}$, uma vez que são observada diminuição dos sistemas visual, auditivo, vestibular e somatossensorial, além da redução na quantidade de neurônios e diminuição de neurotransmissores, o que contribui para uma maior instabilidade da marcha em idosos $^{5}$.

Além das alterações relacionadas ao equilíbrio corporal, controle postural e estabilidade, são também observadas em idosos alterações na velocidade da marcha, uma habilidade motora profundamente complexa na qual os membros inferiores realizam uma série de movimentos cíclicos que acarretam o deslocamento do corpo ${ }^{6}$. A velocidade da marcha tem sido reportada como um componente importante na independência de idosos, estando também relacionada aos riscos de quedas e mortalidade ${ }^{7}$.

A queda é um episódio multifatorial e acidental, limitante e frequente, que pode gerar morbidez, institucionalização, hospitalização ${ }^{8,9,10}$, diminuição da autoeficácia, insegurança e o medo de cair ${ }^{8}$. Este último está entre as ocorrências mais universais das quedas em idosos e pode fazer com que idosos evitem alguns tipos de atividades, o que gera limitações funcionais ${ }^{11,12}$.

No sentido de reduzir efeitos deletérios do envelhecimento e consequentemente fatores de risco predisponentes a quedas, o exercício físico tem sido recomendado, estando este relacionado à melhora da capacidade funcional, equilíbrio, agilidade, coordenação e força muscular ${ }^{13,14}$. Apesar disto, são incipientes estudos que avaliaram o medo de cair em idosos participantes de programas de exercício físico supervisionado. Sendo assim, o presente estudo teve como objetivo analisar o medo de quedas em idosas praticantes de exercícios físicos e em sedentárias.

\section{METODOS}

Este é um estudo experimental transversal, que avaliou 44 mulheres idosas divididas em dois grupos: Grupo A ( $n=22)$ composto por idosas participantes de um programa de exercícios terapêuticos supervisionados desenvolvidos na FCT-UNESP - Núcleo Morumbi na cidade de Presidente Prudente/SP, os exercícios foram aplicados por alunos do curso de Fisioterapia e equipe do setor de pós-graduação em Geriatria e Gerontologia. Grupo B ( $n=22)$ composto por idosas sedentárias que declararam não realizar nenhum tipo de atividade física na avaliação, recrutadas através da mídia; rádio, internet e panfletagem.

Foram incluídas mulheres com idade igual ou superior a 60 anos, que anuíram ao termo de consentimento livre e esclarecido, não foram incluídas mulheres que apresentaram alterações ortopédicas graves, histórico de procedimento cirúrgico recente, alterações neurológicas e sensoriais que pudessem interferir na aplicação das avaliações motoras.

Todas as avaliações foram realizadas em sala em sala ampla, arejada, com boa iluminação e acessibilidade. Inicialmente, os voluntários foram identificados quanto ao nome e idade. Em seguida, responderam uma única vez, das seguintes escalas: Montreal Cognitive Assessment (MoCA), Falls Efficacy Scale (FES-I-Brasil) e o teste de Tinetti. 
A Montreal Cognitive Assessment (MoCA) é utilizada para avaliar a capacidade cognitiva dos participantes, com tempo de administração de aproximadamente 10 a 15 minutos. A pontuação máxima é de 30 pontos, sendo o escore de 26 pontos ou mais é considerado normal; 22 presença de comprometimento leve, 16 tipo de demência de Alzheimer, porém se a pessoa tiver 12 anos ou menos de escolaridade formal, 1 ponto é adicionado como uma correção ${ }^{15}$.

A Falls Efficacy Scale (FES-I-Brasil) foi desenvolvida para idosos, com um questionário que avalia o medo que o indivíduo tem de cair durante o desempenho de 16 atividades. $O$ tempo de aplicação é de 10 minutos, os escores variam de 16 pontos (ausência de medo) a 64 pontos (medo extremo) ${ }^{16}$.

O Teste de Tinetti avalia o equilíbrio e as alterações decorrentes da marcha. Apresenta composição de 16 itens, sendo 9 itens para o equilíbrio corporal e 7 para a marcha. Possui variação de 0 a 1 ou de 0 a 2 pontos, na qual quanto menor a contagem, menor é a capacidade de equilíbrio ou marcha. A pontuação total se dá pela soma da pontuação dos itens, tendo para a marcha a pontuação máxima 12 pontos, para o equilíbrio 16 pontos e de 28 pontos para o total do teste ${ }^{17}$.

Os procedimentos do estudo foram aprovados pelo Comitê de Ética em Pesquisa da FCT/UNESP CAAE: 57835816.5.0000.5402. Todos os voluntários foram devidamente informados sobre os procedimentos e objetivos deste. Após concordarem, assinaram um termo de consentimento livre e esclarecido.

Para a comparação da idade e das variáveis obtidas por meio das escalas MoCA, FES-IBrasil, e Tinetti entre os grupos, inicialmente foi testada a normalidade dos dados por meio do teste de Shapiro-Wilk, sendo aceita a distribuição normal para todas as variáveis descritas. Em seguida foi aplicado o teste t de Student para dados não pareados. Foram consideradas diferença estatisticamente significantes quando o " $p$ " foi menor que 0,05 . As análises foram realizadas por meio do software GraphPad Prisma 5.

\section{RESULTADOS}

Foram avaliadas 44 mulheres divididas em dois grupos; o Grupo A apresentou média de idade de $68,04 \pm 5,3$ anos e o Grupo B 72,54 $\pm 8,4$ anos. Na Tabela 1 observa-se o escore médio dos instrumentos aplicados e com seus respectivos desvios padrão.

Tabela 1. Valores em média, desvios padrão, intervalo de confiança $(95 \%)$ e $p$-valor $(<0,05)$ da idade e escalas MoCA, FES-I-Brasil e Tinetti encontrados nos Grupos A e B.

\begin{tabular}{lllll}
\hline Instrumento & Grupo $A(\mathrm{n}=22)$ & Grupo B $(\mathrm{n}=22)$ & I.C $(95 \%)$ & Valor de $\mathrm{p}$ \\
Idade (anos) & $68,04 \pm 5,3$ & $72,54 \pm 8,4$ & $-8,80$ a $-0,19$ & $0,0427^{*}$ \\
MoCA & $28,31 \pm 1,5$ & $24,45 \pm 1,8$ & 2,82 a 4,90 & 0,4402 \\
FES-I-Brasil & $20,09 \pm 3,7$ & $37,72 \pm 8,7$ & $-21,75$ a $-13,52$ & $0,0003^{*}$ \\
Tinetti & $27,77 \pm 0,5$ & $18,40 \pm 3,9$ & 7,65 a 11,07 & $\mathrm{P}<0,0001^{*}$ \\
\hline
\end{tabular}

${ }^{*} p<0,05$ para Grupo A vs Grupo B. O Escore das escalas foram: MoCA (Montreal Cognitive Assessment $=0$ a 30; FES-IBrasi (Falls Efficacy Scale - International) $=16$ a 64 e Tinetti 0 a 28.

Verificou-se que houve diferença significativa na média da idade das participantes $(p=0,0427)$ e na avaliação cognitiva pelo $\operatorname{MoCA}(p=0,4402)$. Na análise da variável equilíbrio e marcha verificada pelo teste Tinetti, foi encontrada diferença significativa a favor da pratica de exercício físico, sendo $p<0,0001$. Por fim, a análise do FES-I-Brasil, com objetivo de verificar o medo de quedas em idosos, também apresentou resultado favorável para o grupo ativo $(p=0,0003)$, sugerindo que a pratica regular de atividade física atua de forma positiva em idosas. 


\section{DISCUSSÃO}

No presente estudo encontramos menor preocupação com a ocorrência de quedas e melhor equilíbrio e marcha nas pacientes ativas em relação às idosas sedentárias. Estudos realizados com propósito de verificar a funcionalidade de mulheres na terceira idade que sofrem ou não quedas e sentem ou não preocupação com a possibilidade de cair, são interessantes por conceder a possibilidade de traçar o perfil desta população, e com isso produzir informações que colaboram com os fisioterapeutas e equipe interdisciplinar, na criação de estratégias para prevenir quedas e suas consequências na capacidade funcional dos acometidos ${ }^{11,12}$.

A preocupação com a possibilidade de cair é um problema influenciado por diversos fatores, sejam eles ambientais, psicológicos ou físicos. Um estudo realizado em 2015 mostrou evidências de que idosas que sofrem quedas tem menor aptidão funcional, principalmente em relação à agilidade, força muscular e equilíbrio ${ }^{12}$. Em contra partida, a prática de exercício físico é capaz de contribuir para melhora da capacidade funcional, equilíbrio, agilidade e coordenação, qualidade de vida e força muscular, bem como reduzir fatores de risco que podem levar a quedas $^{13,14}$.

O presente estudo corrobora com os dados acima descritos, onde se pode observar equilíbrio e marcha, significativamente melhores em idosas que participam de exercícios terapêuticos supervisionados quando comparado a idosas sedentárias, confirmando assim que os exercícios físicos tem efeito benéfico no equilíbrio das participantes.

Os valores adquiridos na análise do instrumento FES-I-Brasil apontam que as praticantes de exercícios terapêuticos supervisionado apresentam preocupação em relação a quedas significativamente reduzida. O presente estudo corrobora ainda com outros autores, que relacionaram a autopercepção de saúde com o medo de quedas em idosos praticantes de atividade física, que demonstrou melhor percepção de saúde e menor preocupação com a possibilidade de ocorrer quedas em idosos ativos ${ }^{17}$.

$\mathrm{Na}$ literatura, um menor desempenho físico tem sido relacionado ao maior medo da possibilidade de cair ${ }^{5}$, neste sentido, um melhor desempenho físico promovido pela pratica regular de exercício físico pode contribuir de maneira importante na redução do medo de cair apresentado por idosos. Além disto, deve-se também considerar a maior sensação de segurança, mesmo em ambientes desfavoráveis para a estabilidade e equilíbrio corporal, promovida pela pratica regular de exercício físico? .

Apesar dos diversos aspectos positivos ressaltados, o presente estudo apresenta algumas limitações no que se refere ao tamanho amostral, faixa etária heterogênea, que somados puderam influenciar nos resultados apresentados. Como sugestão para futuros estudos, tais apontamentos deverão ser considerados para obtenção de resultados mais concisos.

Conclui-se, portanto que as idosas participantes de exercícios terapêuticos supervisionados apresentam menor preocupação com a possiblidade de cair e melhores níveis de marcha e equilíbrio, quando comparado a idosas sedentárias. Portanto, ser ativo fisicamente parece atuar como um fator de proteção contra o medo de cair e ocorrência de quedas, porém, a realização de estudos de seguimento é incentivada para evidenciar estatisticamente esse efeito.

\section{CONFLITO DE INTERESSE}

Os autores declaram não haver qualquer potencial conflito de interesse que possa interferir na imparcialidade deste trabalho científico.

\section{REFERÊNCIAS}

1. Instituto Brasileiro de Geografia e Estatística - IBGE. Censo Demográfico 2010. Rio de Janeiro: IBGE; 2010. 
2. Netto PM. Tratado de gerontologia. 2. ed. São Paulo: Atheneu; 2007.

3. Hauser E, Sandreschi FP, Parizzotto D, Araújo RCC, Mazo ZG. Medo de cair e desempenho físico em idosos praticantes de atividade física. Rev. Educ. Fís/UEM. 2015; v. 26, n. 4, p. 593-600. DOI: http://dx.doi.org/10.4025/reveducfis.v26i4.28442.

4. Pinho T, Silva A, Tura L, Silva MA, Gurgel S, Smith A, et al. Avaliação do risco de quedas em idosos atendidos em Unidade Básica de Saúde. Rev Esc Enferm USP. 2012; 46 (2):320-7. DOI: http://dx.doi.org/10.1590/S0080-62342012000200008.

5. Perracini RM. Funcionalidade e envelhecimento. Rio de Janeiro: Guanabara Koongan; 2009.

6. Perry J. Análise de marcha: marcha normal. São Paulo: Manole; 2005.

7. Tools A, Rosendahl E, Lundin-Olsson L, Nordstrom P, Gustafson Y, Littbrand H. Usual gait speed independently predicts mortality in very old people: a population-based study. J Am Med Dir Assoc. 2013; 14(7):529. DOI: http://dx.doi.org/10.1016/i.jamda.2013.04.006.

8. Lopes BS, Ribeiro RS, Barros VS, Lopes MEIA, Gomes L, Moraes CF, et al. A relação do medo de cair com eventos de quedas recentes e antigos. Revista Kairós Gerontologia. 2016; 19(1), pp. 47 58. ISSN 1516-2567. ISSNe 2176-901X. São Paulo (SP), Brasil: FACHS/NEPE/PEPGG/PUC-SP.

9. Anjos FR, Gonçalves AK, Griebler EM, Hauser E, Fraga RB, Benin I, Gonçalves BÂJG, et al. Probabilidade de cair e medo de quedas após oficina de equilíbrio em idosos praticantes de atividade física. Revista de Atenção à Saúde. 2015; v. 13, no 44, p.5-10. DOI: http://dx.doi.org/10.13037/ras.vol13n44.2725.

10. Soares WJS, Moraes SA, Ferriolli E, \& Perracini MR. Fatores associados a quedas e quedas recorrentes em idosos: estudo de base populacional. Revista Brasileira de Geriatria e Gerontologia. 2014; 17(1), 49-60. DOI: http://dx.doi.org/10.1590/S1809-98232014000100006.

11. Valduga R, Lopes BS, Farias DL, Nascimento DC, Vieira DCL, Valduga LVA, et al. Risco de quedas e sua relação com a funcionalidade e medo de cair em idosos. R. bras. Ci. e Mov. 2016; 24(1): 153166. DOI: http://dx.doi.org/10.18511/0103-1716/rbcm.v24n1p153-166.

12. Vitor PRR, Oliveira ACK, Kohler R, Winter GR, Rodacki C, Krause MP. Prevalência de quedas em mulheres idosas. Acta Ortop Bras. [online]. 2015; 23(3):158-61. DOI: http://dx.doi.org/10.1590/1413-78522015230300816.

13. Costa VSL, Sousa MN, Alves GA, Alves BVAF, Araújo FR, Nogueira SM. Analise comparativa da qualidade de vida, equilíbrio e força muscular em idosos praticantes de exercício físicos e sedentários. Revista Faculdade Montes Belos (FMB). 2014; v. 8, n 3, 2015, p (61-179), ISSN 18088597.

14. Silva A, Almeida MJG, Cassilhas CR, Cohen M, Peccin SM, Tufik S, et al. Equilíbrio, Coordenação e Agilidade de Idosos Submetidos à Prática de Exercícios Físicos Resistidos. Rev Bras Med Esporte. 2008; Vol. 14, No 2. DOI: http://dx.doi.org/10.1590/S1517-86922008000200001. 
15. Paulo RAF. Montreal Cognitive Assessment (MoCA): Estudo de validação no Défice Cognitivo Ligeiro Vascular. [dissertação]. Coimbra; Faculdade de Medicina da Universidade de Coimbra, 2013.

16. Flávia FOC, Rosângela CD, João MDD, Maria TFF. Cross-cultural adaptation and evaluation of the psychometric properties of the Falls Efficacy Scale-International Among Elderly Brazilians(FESI-BRAZIL). ISSN 1413-3555. Rev Bras Fisioter. 2010; v. 14, n. 3, p. 237-43. DOI: http://dx.doi.org/10.1590/S1413-35552010000300010.

17. Alfieri FM, Silva NOV, Kutz NA, Oliveira MMHA. Relações entre equilíbrio, força muscular, mobilidade funcional, medo de cair e estado nutricional de idosos da comunidade. Revista Kairós Gerontologia. 2016; 19(2), pp. 147-165. ISSNe 2176-901X. 